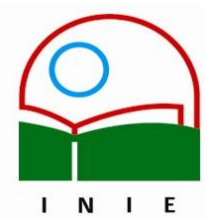

Actualidades Investigativas en Educación

Revista Electrónica publicada por el

Instituto de Investigación en Educación

Universidad de Costa Rica

ISSN 1409-4703

http://revista.inie.ucr.ac.cr

COSTA RICA

\title{
EL ENFOQUE HISTÓRICO CULTURAL EN EL ESTUDIO DEL DESARROLLO HUMANO: PARA UNA PRAXIS HUMANISTA
}

CULTURAL HISTORICAL PARADIGM IN STUDIES ABOUT HUMAN DEVELOPMENT: AN APPROACH TO PRAXIS

\author{
Volumen 9, Número Especial
}

pp. 1-23

Este número se publicó el 15 de noviembre 2009

Gloria Fariñas León

La revista está indexada en los directorios:

LATINDEX, REDALYC, IRESIE, CLASE, DIALNET, DOAJ, E-REVIST@S,

La revista está incluida en los sitios:

REDIE, RINACE, OEI, MAESTROTECA, PREAL, HUASCARAN, CLASCO 


\title{
EL ENFOQUE HISTÓRICO CULTURAL EN EL ESTUDIO DEL DESARROLLO HUMANO: PARA UNA PRAXIS HUMANISTA CULTURAL HISTORICAL PARADIGM IN STUDIES ABOUT HUMAN DEVELOPMENT: AN APPROACH TO PRAXIS
}

\author{
Gloria Fariñas León ${ }^{1}$
}

\begin{abstract}
Resumen: Muchos investigadores dedicados al campo de la educación han realizado estudios en los últimos años basándose en los conceptos del enfoque histórico cultural. Desafortunadamente en muchas ocasiones conocen pobremente las principales ideas de Vigotsky y predominan entre ellos una visión simplista acerca de las dinámicas humanas que propicia entre los investigadores una visión monolítica a la vez que conformista sobre estos temas. En el presente artículo, analizo críticamente las tergiversaciones principales, que a mi juicio, ha sufrido el enfoque histórico cultural y sus causas. Por otra parte, razono la esencia humanista y compleja del paradigma histórico culturalista y su valor explicativo, lo que tiene que ver con las incomprensiones tratadas aquí. El positivismo evade de cierta forma el estudio de los fundamentos teóricos de los enfoques. Mi objetivo fundamental es esclarecer la lógica vigotskyana (consistente en un punto de vista dialéctico complejo en oposición con la filosofía positivista). Finalmente, valoro una investigación que considero como uno de los mejores ejemplos de la visión histórico culturalista sobre la educación y el desarrollo. Enfatizo dos conclusiones fundamentales del análisis: Primero: la tarea principal que tiene un lector para comprender la obra de un autor en el ámbito del desarrollo humano -como es el caso de Vigotsky-, es comprender su axiología y epistemología. En segundo lugar, que ese razonamiento permite conocer verdaderamente el fundamento en que se sustentan las categorías y principios asumidos. Ambos pasos están ligados indisolublemente. El lector tiene que considerar la idiosincrasia del autor, su modo de pensar y su carácter, desde el comienzo de la lectura.
\end{abstract}

\section{Palabras clave: ENFOQUE HISTÓRICO CULTURAL VERSUS POSITIVISMO, CONCIENCIA DE LOGOS}

\begin{abstract}
A variety of investigators, in the last years have proposed researches within the fields of education and human development, based on the concepts of cultural historical paradigm in psychology. Unfortunately in many cases they know very little about Vigotsky's main ideas. Simplistic and linear interpretations about human dynamics (positivist approach), no longer occasion monolithic conformity. This paper deals, on the one hand, with a critical analysis of those misunderstandings, and on the other hand, with a discussion about humanistic and complex essence of the cultural historical paradigm, and even its illumination value. Positivism in human studies frequently keeps thinking away from grounded theory, practice that gives rise to central misunderstandings, I identify, in the present essay. Clarify vigotskyan logos about human development (based on a dialectical and complex standpoint, in opposition to positivistic philosophy) is my immediate aim. Finally, I point up a research, considered as one of the best examples of cultural historical standpoint in education and human development. To finish I stress, two conclusions: First of all, understanding author's epistemology and axiology -in this case, vigotskyan- in human domains, is the reader's major task to deal with. Second, that reasoning provides the understanding about the grounds on which are constructed theoretical categories and principles. Both steps are intrinsically jointed. The reader must consider author's idiosyncrasy, its way of thinking and character, from the very beginning in reading.
\end{abstract}

Key words: CULTURAL HISTORICAL APPROACH VERSUS POSITIVISM, CONSCIOUSNESS, LOGOS

\footnotetext{
1 Doctora en Ciencias Psicológicas en la Universidad Estatal Mijail Lomonosov, Moscú. Doctora en Ciencias de la Universidad de La Habana, Cuba. Actualmente se desempeña como Coordinadora de maestría en Psicopedagogía (la maestría tiene la categoría de Excelencia), Profesora Principal de Psicología Educativa en la Facultad de Psicología de la Universidad de La Habana. Vicepresidenta de la Cátedra L. S. Vygotski (desde su fundación) de la Facultad de Psicología, Editora de la Colección de libros de la Cátedra L. S. Vigotsky. Dirección electrónica: gloria@rect.uh.cu
}

Artículo recibido: 3 de junio, 2009 Aprobado: 19 de octubre, 2009 


\section{INTRODUCCIÓN}

¿A quién podemos considerar propiamente un autor histórico culturalista? es una pregunta que no tiene una respuesta terminante, pues son múltiples y disímiles los matices del pensamiento de uno u otro autor, incluyendo los del propio Vigotsky en diferentes momentos de su obra. No obstante, con conciencia de que el debate quedará abierto, he decidido adentrarme en el tema para dejar mayor constancia de mis apreciaciones acerca de la esencia del enfoque, pues considero que, al margen de las variaciones posibles entre autores, hay un núcleo invariante en su interpretación del desarrollo humano. Conversaré entonces acerca de las dimensiones epistemológica y axiológica en la obra de ese autor. No me quedaré en el análisis teórico, procuraré también de alguna forma, sus derivaciones para el diseño de la educación.

Con frecuencia se estima que seleccionar como fundamento central de una investigación el concepto de zona de desarrollo próximo, el de mediación u otros términos, nos convierte, automáticamente, en un autor de esa estirpe. Sin embargo, esto debe analizarse con mayor detenimiento. Utilizo el concepto de mecanismo de defensa, pero esto no me hace psicoanalista, solo significa que respeto los aportes del psicoanálisis, que los refiero cuando es oportuno. $Y$ es que cada enfoque, además de la terminología tiene un sentido particular que se manifiesta como su ánima teórica. Tanto la terminología como el espíritu de una obra tienen valor, pero a la hora de decidir su preeminencia me pronuncio por el segundo. A mi juicio son los valores $-y$ con ellos el espíritu- los que tratan de canalizarse a través del instrumental conceptual para definirse más claramente, no tanto a la inversa. Por esta razón, jerarquizaré, en primer lugar, la exposición del espíritu de la obra histórico culturalista desde mi perspectiva y dejaré en un segundo plano la letra.

Inicialmente, procuraré la identificación del estilo en la epistemología y en la axiología del enfoque. Me atendré en especial al pensamiento vigotskyano, aunque es muy difícil no hacer alguna que otra referencia al de sus seguidores más directos, ya que ellos intentaron contestar las cuestiones que su maestro les dejara planteadas. Más tarde, reflexionaré sobre su denominación como enfoque histórico y cultural. Pondré ejemplos tomados de fuentes que me parecen mostrar de forma más fiel lo emblemático de este pensamiento. Para terminar, analizaré problemas que pienso debieran ser resueltos de alguna forma, para que el enfoque no se anquilose y logre evolucionar más allá de la ortodoxia. Considero que vale 
mantener viva esta lógica de pensamiento, atemperada a las demandas de la civilización actual, la propia naturaleza histórica y cultural del enfoque lo reclama. No enumeraré las características que creo distintivas, me propongo captar su alma, pues estimo que en esto radica, en cierto sentido, el problema que deseo tratar. Me toparé repetidas veces con una dificultad, que es tal vez una virtud del enfoque: al ser complejo y dialéctico, no se puede plantear su contenido como un índice de tópicos o aspectos, pues todo está tan interconectado, que es difícil destacar algo sin tratar lo restante. A pesar de esto, he concebido ese orden, que procuraré seguir todo el tiempo, pero estaré muchas veces en varios puntos a la vez. Trataré de evitar el peso de la construcción lógico-semántica que Mecheriakov (1996) presentó con tanto detalle, para concentrarme más en el carácter del enfoque.

\section{Conciencia de logos}

La fundación del enfoque exigió a Vigotsky el estudio crítico y sistemático del pensamiento de la época -no solo psicológico-, así la intención de su necesaria superación. Su obra no fue una concepción teórico metodológica derivada por simple inducción a partir de los hechos de la práctica, aunque sus fundadores tomaron esta como exigencia mayor. El ensayo "El sentido histórico de la crisis de la psicología" constituye su plataforma conceptual crítica. Sus enunciados son reiterados sistemáticamente por Vigotsky (1982a) en la elaboración teórico metodológica de toda su obra. Esta enuncia la necesidad del planteamiento revolucionario del conocimiento científico y de sus herramientas para la intervención en el desarrollo humano. A mi juicio, el enfoque histórico cultural se funda a principios del siglo $\mathrm{XX}$, como una psicología crítica. Mantener consciente la lógica del pensamiento que tomaba cuerpo fue finalidad clara del documento. No perderla en la actualidad, significa respetar su linaje.

El estatuto propuesto en ese documento, da una visión compleja y dialéctica sobre la psicología, (el desarrollo de la personalidad y su condicionamiento) ${ }^{2}$, superando los planteamientos unilaterales, reduccionistas e hiperbolizantes sobre este tipo de procesos y fenómenos, presentes en las otras concepciones. Vigotsky plantea como necesaria la

2 No fue por gusto que el primer debate sostenido en la Cátedra "L. S. Vigotsky" (1998) de la Facultad de Psicología de la Universidad de La Habana que para su fundación tratara precisamente esta obra. Volumen 9, Número Especial, Año 2009, ISSN 1409-4703 
mirada multilateral e integradora sobre el ser humano, concebido en devenir, a la vez que encajado en un entorno cultural cambiante a lo largo de la historia, donde el sujeto obra su personalidad amalgamando un naturaleza biológica y social -única e irrepetible- en mancomunidad con los Otros. Con esto, el enfoque histórico cultural se aparta de la óptica abstracta $^{3}$ acerca del ser humano, pues sin perder el estudio de las regularidades -lo general-, busca la originalidad del sujeto -lo singular- en el momento histórico y cultural que este vive.

Su punto de vista es determinista, pero complejo y dialéctico, sin lugar a causalidades mecánicas. El materialismo en él, no es vulgar. La determinación principal: la educación como expresión de la cultura-, no es concebida linealmente. Ella tiene determinado grado de entropía. Apuntaba Vigotsky destacando la dinámica dialéctica de sus condicionantes (externos-internos, sociales-individuales): “...En última instancia, el niño se educa a sí mismo. En su organismo, y no en cualquier otro lugar, transcurre la lucha decisiva de las diferentes influencias que definen su conducta por muchos años..." (2001, p. 113).

La conciencia de su logos encontró múltiples expresiones metafóricas en la obra de Vigotsky, ya que gustaba de utilizar alegorías para representar el giro que debía dar el pensamiento del psicólogo. Creo que las utilizaba, a fin de esclarecer mejor la índole y envergadura del enfoque histórico cultural, en la comprensión y explicación sobre los fenómenos y procesos del desarrollo humano. Una de las mejores metáforas es la del agua. Esta ilustra la unidad dialéctica del objeto de estudio, por ejemplo, en sus dimensiones cognitivo-afectivas: la vivencia, que constituye la piedra angular para comprender el aparato teórico metodológico propuesto. Es decir, el agua es a la vivencia, como el oxígeno y el hidrógeno, por separado, son a lo cognitivo y a lo afectivo. Su idea sobre las unidades complejas descansa en la asunción de esta unidad dialéctica de lo externo-lo interno, lo afectivo-lo cognitivo que es la vivencia. Al respecto decía Vigotsky que: “...en la ciencia,...el análisis de los elementos debe sustituirse por el análisis... de una unidad compleja..." (1987, p. 7). Esto incita a la búsqueda de modos de trabajo e indicadores que expresen dicha fusión.

3 En este enfoque se consigue intencionadamente la visión de lo general a través de lo singular y viceversa. 
Otra metáfora es la de la "geología" de la mente humana, los estratos que se "fosilizaron" en esta a lo largo de la historia de la especie humana (antropogénesis) o los que se "fosilizan" en el sujeto particular durante la ontogenia. El vocablo fosilización da la idea de la conservación histórica de los procesos, en la constitución de la potencialidad para la realización grupal y personal. Decía al respecto: “...la estructura del desarrollo del comportamiento hace recordar, en cierto sentido, la estructura geológica de la corteza terrestre. Las investigaciones establecieron la presencia genética de distintos mantos en la conducta del hombre. En este sentido, la geología de la actuación humana...”(1987, p.151). Esta última frase concreta la manera de ver el desarrollo y de estudiarlo.

La metáfora de la "zona" (en el concepto de zona de desarrollo próximo) -referida al surgimiento de las potencialidades del desenvolvimiento humano, gracias a la acción transformadora del sujeto sobre la realidad en conjunción con la orientación cultural que recibe de los Otros-, es una de las más conocidas.

Para ilustrar mi idea recordaré que, refiriéndose al desarrollo, Vigotsky apuntaba:

...tomemos en atención que dicho desarrollo constituye un proceso dialéctico complejo, que se caracteriza por una periodicidad múltiple, por una desproporción en el desarrollo de las distintas funciones, por las metamorfosis o transformaciones cualitativas de unas formas en otras, por el complicado entrecruzamiento de los procesos de evolución e involución, por la entrelazada relación entre los factores internos y externos y por el intrincado proceso de superación de las dificultades y de la adaptación. (1987, p. 151)

Y representándose el concepto de zona de desarrollo decía:

Igual que el horticultor, que deseando determinar el estado de su huerto, no tendrá razón si se limita a valorar los manzanos que ya han madurado y han dado fruto, sino que debe tener también en cuenta los árboles en maduración, el psicólogo, al valorar el estado del desarrollo, debe tener obligatoriamente en cuenta no sólo las funciones maduras, sino también las que están en trance de maduración. No sólo el nivel actual, sino también la zona de desarrollo próximo. (1982b, p. 130) 
La periodicidad múltiple, las metamorfosis, los momentos de evolución-involución, las relaciones entrelazadas entre los factores internos y externos, el trance de maduración, precisaban no de una expresión puntual sino una expresión espacial más amplia. El punto sería el espacio más reducido, sobre todo si se compara con la zona. A su vez, el espacio era visto como un huerto -segunda metáfora sobre el espacio dentro del mismo concepto-, espacio fértil o espacio a fertilizar. En este caso, la metáfora es menos obvia que en los restantes, pero indudablemente requirió de los recursos del pensamiento metafórico para representar el concepto. Si abundamos en detalle, en tres de las metáforas mencionadas -la tercera es la siguiente metáfora-, Vigotsky recurre de una manera u otra al espacio y lo representa indirectamente como la tierra (las capas geológicas, el huerto abonado y la siembra o enraizamiento, como señalo a continuación).

La cuarta y última metáfora que mencionaré es la de enraizamiento ${ }^{4}$ del ser humano en la cultura. A mi juicio esta tiene como fin la expresión de cuán incorporada debe estar la persona a la cultura, para lograr su desarrollo. Decía Vigotsky: “...la cultura crea formas especiales de conducta, cambia el tipo de la actividad de las funciones psíquicas...El arraigo del niño en la civilización está condicionado por la maduración de...funciones correspondientes" (1987, p. 45). A mi juicio, las metáforas mencionadas, entre otras, transmiten muy bien el espíritu de su obra, el sentido de su teorización: su alma.

En oportunidades, los lectores al fijarse más en las categorías, principios y leyes particulares formulados, que en la lógica general del pensamiento vigotskyano, terminan considerando más la letra que su espíritu. Tanto una lectura como la otra tienen su función en el análisis de cualquier sistema conceptual, pero no pueden desvincularse. Sin embargo, para comprender a fondo la letra hay que estar imbuido de su sentido, de su alma. Con cierta frecuencia los psicólogos y los pedagogos, que tienen propósitos básicamente instrumentales, están más pegados a la letra o terminología específica que al espíritu de la obra. Destacar el Leitmotiv de la obra vigotskyana, su valor -tanto en el plano ético como epistemológico-, me parece la mejor introducción al estudio de su letra, si queremos hacer un análisis profundo de esta.

$4 \quad$ Utilizo indistintamente enraizamiento cultural y enculturación. 
Cada teoría, encierra un logos, un estilo de pensamiento, que debemos asimilar integradamente con su aparato conceptual.

El espíritu del enfoque a mi juicio versa esencialmente sobre la determinación compleja y dialéctica de los procesos del desarrollo del ser humano (en la constitución de su personalidad), encontrada en la cultura y la historia ${ }^{5}$. Su conceptuación, ajustada a la complejidad del desarrollo, mantiene el mismo carácter a través de planteamientos integradores, flexibles y abiertos, sin marcada intención de cristalizar como instrumento acabado de pensamiento. Esto se opone al afán instrumentalista de diferentes autores que hallan el valor de esta obra más en los términos específicos "listos para la práctica", que en la cosmovisión sobre el devenir (historia) del ser humano -como especie e individuo- en la cultura.

Para continuar la lógica del enfoque, propongo analizar a continuación los dos derroteros principales del razonamiento histórico culturalista sobre el desarrollo de la personalidad. Terminaré con apuntes acerca de las implicaciones de esta forma de pensamiento en la práctica.

\section{Lo histórico}

La asunción del tiempo como infraestructura del desarrollo, afina la comprensión y explicación de la dimensión histórica del devenir humano en este enfoque, lo que se expresa -entre otros ejemplos- en el peso atribuido por este a la memoria, en el desarrollo humano. Esta es asumida como memoria histórica, tanto en el plano de análisis sobre sujeto como de la cultura.

En el plano cultural, la memoria histórica se observa como preservadora del patrimonio en forma variada (valor espiritual, sentimiento, concepto, libro, obra pictórica, costumbre, entre otras posibles). En el análisis del desarrollo del sujeto es tomada como su sustrato genético. Ella es considerada, al menos en dos formas típicas. Primeramente, con un sentido más antropológico: “...hacer el lazo para recordar, fue, precisamente, una de las primeras formas de habla escrita..." (Vigotsky, 1987, p. 84). En segundo lugar, con un sentido psicológico,

5 Lo que abarca también como dije anteriormente, la amalgama con lo biológico obrada por el sujeto. Volumen 9, Número Especial, Año 2009, ISSN 1409-4703 
como la dinámica pasado-presente-futuro, por ejemplo, del concepto "zona de desarrollo próximo".

Los estudios típicos del enfoque sobre la memoria, en las tempranas etapas del desarrollo ontogenético, destacan su surgimiento como neoformación primigenia que se integra a las restantes para subyacerlas en la constitución de la personalidad ${ }^{6}$. La memoria permite la fosilización de los restantes procesos de esta. En la siguiente cita se recalca su papel, además de hacerse patente el peso de lo biológico y la amalgama de ambos:

...La organización de la actividad nerviosa superior ... crea la posibilidad de regulación del comportamiento desde fuera...el dominio sobre la memoria de otro hombre se estructura, en principio, de la misma manera, como se ejerce el control sobre la memoria propia... (Vigotsky, 1987, p. 85)

Debo añadir que lo histórico también se aprecia en el tratamiento del maridaje paulatino de todas las neoformaciones surgidas (capas geológicas en la metáfora vigotskyana), a lo largo del desarrollo humano gracias a la memoria. A diferencia de los llamados enfoques holísticos (el humanismo de corte existencial, entre otros), que dejaron de lado el complejo acople de los procesos de la personalidad en el curso ontogenético de sus relaciones (partetotalidad $)^{7}$, el enfoque histórico cultural hace gala de su comprensión y explicación genética, partiendo del estudio de la estructuración histórica -dialéctica y compleja- de dichos procesos. Para Vigotsky la personalidad en desarrollo es el objeto esencial de atención, que refirió como “...Síntesis psíquica superior que...debe ser denominada personalidad...la historia del desarrollo cultural del niño nos conduce a la historia del desarrollo de la personalidad" (1987, p.50).

Siguiendo los postulados vigotskyanos generales se planteó más tarde, una teoría de la periodización sujeta al condicionamiento histórico cultural, en el cual se destaca que el desarrollo cultural de la sociedad señala los derroteros al desarrollo psicológico individual. La historia individual se articula partiendo de las tareas culturales (lo general), que le plantea la sociedad a cada generación (entretejidas con las singulares del sujeto en cuestión),

\footnotetext{
Vigotsky hablaba de personalidad, no así de sujeto.

Viendo aquí los procesos como las partes de la personalidad (la totalidad).

Volumen 9, Número Especial, Año 2009, ISSN 1409-4703
} 
matizadas por la cultura donde se arraiga. El juego, el trabajo, la actividad de estudio, surgidos como necesidad en la historia de la civilización, se convierten en rectores según los fines y tareas culturales para cada edad. La altura del desarrollo de la personalidad se aprecia a partir de las demandas culturales, de las tareas que la sociedad adjudica a los seres humanos en las diversas etapas de la vida. Para alcanzar la altura requerida en cada período del desarrollo humano, es condición para el sujeto apropiarse del patrimonio cultural, como recurso civilizador, porque en este se encuentra cristalizada la espiritualidad ${ }^{8}$ propia de cada época histórica de la humanidad.

Como apunté, en el enfoque se destacan los tres tipos de actividades fundamentales surgidas en la evolución de la sociedad: la actividad lúdica, la actividad laboral, la actividad de estudio, que se engranan histórica y culturalmente, lo que se expresa en las diferentes investigaciones sobre el desarrollo de la personalidad. La historia personal es en parte -sin desubicar del centro a la comunicación-, el acoplamiento paulatino de estas formas concretas de la vida humana. Ellas asumen una configuración singular, a partir de la vivencia de cada sujeto. Gracias a esas actividades en amalgama mutua, y simultáneamente en amalgama con la comunicación, es que el sujeto se inserta en la cultura ora como aprendiz, ora como creador, ora como creador-aprendiz, haciendo patente su estilo, su personalidad $^{9}$. Cuando el sujeto se margina en la sociedad podemos buscar de qué actividad o actividades fue excluido ¿del estudio? ¿del trabajo? ¿de actividad lúdica?, a la par de buscar sus vivencias (desde lo comunicativo): ¿cómo enfrentó esa exclusión, esa agresión? La imbricación histórica entre actividad-comunicación es un punto cardinal del análisis. Ambas dirigidas al enraizamiento cultural y al "dominio de la propia conducta" (Vigotsky, 1987, p. 83).

La comunicación orienta la transformación de la realidad y la actividad la produce efectivamente. "Un hombre influye sobre otro a través del habla" (Vigotsky, p. 95), pero esto no excluye la acción. Ambas se encuentran en perenne interacción. Así fraguó esta unidad en la antropogénesis, cuando comunicación y actividad se encontraron para "fosilizarse" como unidad indisoluble. Ellas son como la cara y la cruz de una misma moneda, cuando

\footnotetext{
Vista como valor, arte, ciencia, técnica, etcétera.

Como he planteado en otros trabajos asumo el concepto personalidad en sus dos acepciones. Como totalidad compleja y como sello único e irrepetible (estilo). Volumen 9, Número Especial, Año 2009, ISSN 1409-4703
} 
una está a la vista no es porque la otra esté ausente, sino porque está sobreentendida. En la obra de Galperin, uno de los discípulos más sobresalientes de Vigotsky, se puede apreciar el itinerario dialéctico de ambas, sus conversiones mutuas durante la formación y desarrollo del plano mental en un sujeto determinado. Es la palabra, el código primordial de interiorización ${ }^{10}$ de la actividad. Y esta, la transformación práctica de la realidad, explícita o implícitamente orientada por la palabra. Las dos constituyen también el tejido de la exteriorización. Comunicación-actividad, se unieron en la historia para no separarse, solo en la enfermedad donde pueden encontrarse disociadas. Ellas son las vías del enraizamiento cultural del individuo.

Con lo histórico se asume lo dialéctico. No se parte ni se llega a un punto fijo sino mutable según las exigencias de la época de la civilización ${ }^{11}$ en que se vive, y estas cambian vertiginosamente en los últimos tiempos. La conceptuación de la "situación social del desarrollo" nos da la clave para reflexionar sobre el condicionamiento del desarrollo. La unidad compleja de la actividad - la comunicación obrada por el sujeto en mancomunidad le da cuerpo a su historia personal. Aunque no todo el desarrollo ha sido estudiado desde esta lógica general por sus seguidores en el mundo, pudiéramos apuntar que es óptica esencial del enfoque.

\section{Lo cultural}

Decía Geertz: “...sin hombres no hay cultura por cierto, pero igualmente, y esto es más significativo, sin cultura no hay hombres" (2003, p. 72). Parto de esta consideración, porque creo que un pensamiento análogo sustentó la denominación del enfoque, como cultural. Pienso que hubieran podido ser palabras de Vigotsky.

Las relaciones entre los seres humanos no son naturales, sino que están mediadas por la cultura. Es una perogrullada afirmar que no es lo mismo la relación padre-hijo, hombremujer, maestro-alumno en occidente que en tierra japonesa. Todas las relaciones humanas están mediadas por la cultura, son relaciones culturales. La cultura actúa como

10 Vigotsky empleó el término vrashivanie y ovnutryvanie, no interioriztatzy.

11 La generación que ha aprendido las NTIC en edades tempranas, tiene ventajas en la apropiación de su valor técnico y determinadas desventajas en relación con la apropiación de otros valores espirituales, respecto a otras generaciones. 
condicionante del desarrollo hasta el punto de determinar la constitución mental del ser humano. Determinación de naturaleza compleja y por ende no lineal, no directa, no inmediata; que contempla los procesos subjetivos emergentes. Su observación es decisiva en la comprensión y explicación de lo humano, como ser o como obra.

Las herramientas culturales concretas o abstractas, son frutos del talento humano, a la par que sus constituyentes. Fue así desde el surgimiento del trabajo, del arte y de la expresión afectiva sutilizada, no animal. Las capacidades humanas se cristalizan en las herramientas de la cultura, al igual que las herramientas de la cultura se cristalizan en las capacidades humanas. Se componen mutuamente. Recuerdo que Engels apuntaba que "... la mano no es solo el órgano del trabajo, también es el producto de él..." (1961, pp. 143-144). Esta cita nos muestra, a través de ese experimento mayor que fue la antropogénesis, como cultura y naturaleza se fundieron para dar un resultado mayormente cultural: la humanización de la mano (el órgano), a través de su enculturación. Sin la creación del instrumento, que simultáneamente se convirtió en patrimonio de la cultura, no hubo mano posible. Decía Vigotsky: “...las funciones naturales conservan su existencia dentro de las culturales ... "el desarrollo del habla... un buen ejemplo de tal unificación... La cultura no crea nada, ella solo transforma las dotes naturales en correspondencia con los fines del hombre" (1987, síntesis del texto de las pp. 98, 40 y 163). Las citas referidas, ilustran -a mi juicio- lo palmario de la cultura en el enfoque y la causa de su peso en su denominación. Lo erróneo en estos casos sería concebir instrumentalmente la cultura. Llamo la atención sobre este asunto, porque el enfoque ha tenido diversas interpretaciones positivistas, nada más lejos de su esencia.

La denominación histórico social, a mi entender, no es muy apropiada, porque las relaciones entre los seres humanos siempre están mediadas por la cultura y contenidas de esta, recordemos las palabras de Geertz. La cultura se impone en ellas, aunque esto no quiere decir que lo natural no pese. Cuando estamos enfermos, cuando sufrimos un cataclismo medioambiental, estamos más a merced de la naturaleza: de lo corporal o del entorno. Pero nuestra asunción de tales males "naturales", es cultural. Sin olvidar que muchos de ellos son en buena medida resultantes de una cultura no racional. En la cita anterior se destacó la importancia de las funciones primarias "naturales" en el desenvolvimiento humano, estas están contenidas en las funciones superiores y constituyen su basamento. 
El médico, quien tiene una relación directa con el cuerpo de sus pacientes, intenta curarlos mediante herramientas culturales que suelen utilizar los recursos de la naturaleza (vacunas, medicamentos, entre otras), pero con un fin cultural: la profilaxis, la cura. La relación médicopaciente es cultural, no es simplemente un nexo natural hombre-hombre $u$ hombre-mujer. Incluso el género y las relaciones entre los géneros se obran culturalmente. No es lo mismo género que sexo. Solo encuentro ejemplos de relaciones culturales entre los seres humanos. El conductismo, el constructivismo y otras aproximaciones al aprendizaje y al desarrollo humano vacían las relaciones interpersonales de su contenido cultural, simplemente ignorando que esta es su esencia.

Estos enfoques no incorporan la cultura y su expresión en la educación, como recurso teórico para la comprensión y explicación del desarrollo humano. Aunque, por supuesto, al existir la educación de manera tan fehaciente para todos, se ven obligados a utilizarla en sus prácticas. Pero no es lo mismo asumirla en la práctica que formularla consecuentemente en teoría-metodología-práctica. Convertirla en praxis requiere coherencia en su formulación desde la teoría. La asunción de la educación en los planos teórico y metodológico, como directora del desarrollo, es privativa del enfoque histórico cultural. Puede haber sido tenida en cuenta más tarde por otras corrientes de pensamiento, pero sin su enunciación de esencia.

La educación, en su sentido amplio, es el recurso primordial para la enculturación de los seres humanos. No es que Vigotsky no hablara de relaciones sociales, claro que lo hizo, es difícil estar pendiente todo el tiempo del hábito cotidiano y tradicional del lenguaje. Sin embargo, su énfasis estuvo en el proceso de enraizamiento del ser humano en la cultura, en la conversión del hombre y la mujer en seres cultos, desde las épocas más tempranas de la vida. En la constitución de las "funciones culturales". En la transformación de las funciones "naturales", gracias al refinamiento cultural. El ser humano, nace como ser social, quiere decir que de esta condición no se le puede alienar, porque ya es suya al nacer. En cambio, el acceso a la cultura depende en buena medida, de las oportunidades al alcance de la persona. Por tanto, se puede marginar al ser humano de la cultura, no de su condición social. Estimo que esta es una de las razones posibles de su énfasis en lo cultural. 
La no consideración del papel determinante de la educación en el desarrollo humano -en su sentido complejo dialéctico-, acerca cualquier enfoque más al conductismo, al cognitivismo y a otros esquemas de pensamiento similares, que al enfoque histórico cultural, pues esta es una de sus consideraciones sine qua non ${ }^{12}$. Pero como ya dije, la adhesión al instrumentalismo en la educación, no asegura su valor de esencia en la concepción del desenvolvimiento humano ${ }^{13}$.

En consecuencia, es imprescindible en cualquier análisis o experiencia que se precie de ser histórico culturalista, la consideración de los cánones culturales. Vigotsky decía: "Al cambiar por completo el tipo de adaptación en el hombre, aparece en primer plano el desarrollo de sus órganos artificiales: los instrumentos...” (1987, p. 35). Y añadía más tarde:

La utilización de medios auxiliares ...reestructura en su raíz toda la operación psíquica, análogamente a la forma en que la utilización de instrumentos modifica la actividad natural de los órganos y amplía, hasta el infinito, el sistema de activación de las funciones psíquicas. A una y otra cosa la denominamos...función psíquica superior... (1987, p. 103)

Rogers, y otros tantos que hablaron de la educación y desde la educación, escribió un libro fabuloso sobre la educación (1993), pero esto no lo hace histórico culturalista, o culturalista simplemente. Todo depende de cómo se asuma la educación, y su lugar como categoría o principio teórico metodológico -explicativo o comprensivo-, además de su expresión como herramienta de trabajo profesional. Los psicólogos tendríamos que reconocer clara y públicamente nuestros verdaderos compromisos filosóficos y políticos, aquellos que nos mueven tácita o expresamente. Porque en psicología, todo está vinculado, de alguna manera, a ambas: filosofía y política. La estimación de la educación en la elaboración teórico metodológica del cuerpo conceptual de una obra, es uno de los mejores ejemplo de tales compromisos, por demás éticos. Un estado puede dar mayor o menor peso a la educación a

12 Estas teorías asocian el desarrollo al aprendizaje, salvo el conductismo que los iguala. Debemos captar la sutileza de esta diferencia, ya que en ella encontramos condicionantes ideológicos del problema, que sin referirme al término aprendizaje, trataré después.

13 Es esta la razón, de mi interpretación en el libro "Psicología, educación y sociedad", acerca del papel de la educación como categoría, en la construcción teórica de cualquier modelo sobre el desarrollo humano, ya pertenezca a los estancos establecidos tradicionalmente de: la psicología educativa, social, clínica, organizacional y otros posibles campos en los que se estudie o promueva dicho desenvolvimiento. Volumen 9, Número Especial, Año 2009, ISSN 1409-4703 
través de inversiones reales para su mejoramiento, mientras que una teoría psicológica puede darle también mayor o menor peso en su determinación del desarrollo humano, para así servir de justificación a una u otra política-, aunque no se lo proponga explícitamente. La ciencia no es aséptica y menos la psicología que parte de las necesidades y las aspiraciones humanas. El enfoque histórico cultural toma la cultura, y su expresión en la educación, como determinante complejo, como condición.

J. Bruner, por ejemplo, se reconoce a sí mismo como culturalista, no histórico culturalista a pesar del conocimiento que tiene de este sistema teórico. Considerar la narrativa y su relación con la cultura, no lo convierte automáticamente en un ejemplo de este modo de pensar, aunque la narrativa tiene un curso histórico. Su libro "La educación a las puertas de la cultura" (1997), a pesar de su proximidad a las consideraciones vigotskyanas, no las incluye, aunque por supuesto, está más cerca de estas que otros autores. Pero, mientras no tome lo histórico y lo cultural en el sentido apuntado, como esencia, no puede tildársele de tal. En cambio la posición V. V. Davidov en su ensayo "La educación que desarrolla en la escuela del desarrollo" sí lo es. En Davidov ${ }^{14}$, la educación no está a las puertas de la cultura, sino organizada a los fines del enraizamiento cultural.

Otra razón importante para llamar histórico cultural al enfoque es que su fundador así lo denominó. Quisiera agregar a esto que la calificación de histórico cultural puede ser más inclusiva. Siguiendo la lógica apuntada por Geertz que comparto, lo cultural incluye lo social en este enfoque, pero no a la inversa. Se trata de una regla lógica sencilla: un ser humano puede existir sin ser culto, pero si es un ser culto, es humano. Un sujeto al ser excluido de la cultura, deja de ser considerado como persona en su sentido ciudadano. Es un marginal, condenado de cierta forma, a la escala inferior del ser. De crecer como ser social nadie se libra, porque se nace siendo humano. Es decir, se nace siendo social. Se nace preparado para asimilar la cultura de los afectos-conocimientos-valores, a través de su relación con otros sujetos. El ser humano en solitario "sigue funcionando en comunión". Cuando el ser

14 Su trabajo va mucho más allá de lo apuntado por mí hasta aquí, aunque de cierta forma lo contiene, ya que está dedicado al planteamiento de la plataforma de ideas para la reforma de la educación contemporánea. 
humano muere, sigue viviendo a través de lo que legó a la cultura: su obra, por local o pequeña que esta sea. Su acción queda así en la memoria histórica.

Considero que hay una aproximación naturalista a los problemas del desarrollo humano, cuando se le vacía de cultura, cuando se habla de relaciones humanas al margen de la interdependencia compleja que existe entre educación-cultura-enculturación-desarrollo de la personalidad. Cuando ella no se enuncia con diafanidad en el encuadre teórico metodológico de una concepción sobre el ser humano.

El planteamiento del enfoque vigotskyano, como histórico social, pudiera servir a los fines de posiciones en cierta forma no muy progresistas; como aquellas que culpan a los marginales de su situación social, como si no hubiera diferencia de oportunidades en el acceso a la cultura, en la sociedad actual a escala mundial. El condicionante en este caso no es la sociabilidad (o la no sociabilidad), sino la cultura o la incultura. Ser cultos para ser libres, decía José Martí, pues hay que serlo para conseguir el pleno desarrollo de la personalidad. La sociabilidad no se conquista, se nace con ella. Es la oportunidad de acceder a la cultura, a la educación durante toda la vida, la que nos permite desarrollarnos sostenidamente cultivando el ser social.

Lo histórico y lo cultural fueron planteados aquí separadamente solo con fines didácticos. Historia y cultura están intrínsecamente unidas. Pudiéramos decir que la historia no es otra cosa que: la historia de la civilización o de la incivilidad. El análisis del complejo condicionamiento histórico cultural del desarrollo humano a través de la educación (implica la autoeducación como condición intrínseca), es la vía para penetrar en la comprensión y explicación de sus procesos, formaciones y características. Manera de ir del fenómeno a la esencia, de no quedarse en la superficie de la observación, de ir del examen "fenotípico" al examen "genotípico", tal como Vigotsky los nombraba, en su hábito de metaforizar.

\section{La práctica}

No hay mejor ejemplo de la conjunción de lo histórico cultural en la concepción analizada que las consideraciones sobre la educación en la génesis del desarrollo de la personalidad. La educación es el recurso principal de la cultura, a los fines del enraizamiento cultural, del Volumen 9, Número Especial, Año 2009, ISSN 1409-4703 
dominio del comportamiento propio. Es en ella, gracias a ella, que se genera el desarrollo. Recordemos que: “...Cualquier función en el desarrollo cultural del niño aparece en escena dos veces, en dos planos; primero como algo social, después como algo psicológico; primero entre la gente, como una categoría interpsíquica, después, dentro del niño, como una categoría intrapsíquica” (Vigotsky, 1987, p.161).

En otras palabras, esa relación interpsíquica es esencialmente educativa. Vigotsky decía que: "El mismo proceso de educación siempre se realiza en forma de cooperación...y representa un caso específico de interacción de formas ideales y existentes..." (1982b, p.14). Y especificaba más adelante algo crucial, que no siempre es considerado por los estudiosos más nuevos del enfoque: “...toda educación constituye una fuente de desarrollo, pero no toda educación es buena, solo la que adelanta el desarrollo..." (1982b, p.14). Es decir, que la educación debe cumplir determinados requisitos de calidad para provocar tales consecuencias.

Acerca de esta sutil observación investigaron bastante sus seguidores, y es a ella que haré referencia enseguida por considerarla una de las características emblemáticas del enfoque, que además expone claramente el nexo de lo histórico y lo cultural. Uno de los ejemplos, a mi juicio, más auténticamente histórico culturalista es el trabajo de Aidarova (1969 y 1979) sobre el desarrollo de la conciencia del idioma en los niños a través de la enseñanza, que no referiré porque está traducido al menos al inglés. Por otra parte, se encuentra referido en el libro de Davidov (1981) "Tipos de generalización en la enseñanza”, traducido al español. Animo a leerlos. En él se asumen, entre otros requisitos:

> El saber histórico como condición del conocimiento refinado, culto. Quiere decir que para catalogar a una persona como experta o culta en una materia determinada, esta debe conocer el origen o la historia de esa rama del conocimiento. Además, el conocimiento del origen de los distintos fenómenos y procesos refina el pensamiento en tanto lo torna más profundo. La profundidad del pensamiento se asocia al conocimiento de la génesis de las cosas. Un pensamiento superficial, no se considera refinado, no se considera culto. La historia aporta el fundamento, la posibilidad de argumentar lo que se razona. La experiencia de Aidarova remonta los razonamientos al condicionamiento histórico (la aparición de los diferentes signos en la comunicación, 
el origen de las palabras, etcétera), lo que no constituye un razonamiento episódico sino habitual. Esta es la primera capa -al decir de la metáfora sobre la geología de mente-, de los razonamientos que sobrevendrán todo el tiempo durante el aprendizaje.

> La conciencia, siempre crítica, como fruto y condición del desarrollo complejo de la personalidad y como indicador del carácter unitario de la vivencia. Los sujetos del experimento elaboran un relato personal acerca de sus descubrimientos en el idioma, las regularidades de la estructura y funcionamiento del mismo, los puntos críticos, las excepciones y casos curiosos, entre otros aspectos. Igualmente los métodos de razonamiento. Esta conciencia aflora como sensibilidad hacia el idioma, que les permite preciar a los sujetos los fenómenos del mismo.

> La conciencia como resultado personal, creativo del intercambio o la cooperación con los Otros. A las explicaciones anteriores se suma que, a los sujetos se le plantean acertijos de la lengua para resolverlos creativamente, lo cual se sustenta en la combinatoria del trabajo individual-grupal. Los sujetos historian su aprendizaje en la libreta de apuntes "Mis descubrimientos en el idioma", que constituye un verdadero libro de texto, de estos tiempos

> La generalización (y el pensamiento así que proceso de la personalidad), como condición del desarrollo. La generalización en calidad de esencia, del paso de lo fenotípico a lo genotípico, antes que transferencia, que es el modo en que se comprende por otros enfoques. El énfasis en el método de análisis eleva el pensamiento a cosmovisiones sobre la lengua, lo que dota a los sujetos de recursos mentales para descubrir la esencia detrás de los fenómenos del idioma y para recrear la imaginación. La asunción de la creatividad como un indicador del desarrollo abre las posibilidades de influencia sobre el desarrollo de la personalidad, por ser ella la piedra toque de su estructuración.

$>\quad$ La necesidad de la sistematización (reproducción) creativa del conocimiento depositado en la cultura. El sujeto interioriza lo que crea, no interioriza puramente lo creado por los otros. En otras palabras, para interiorizar adecuadamente debe recrear lo creado por los otros. La interiorización no es un proceso lineal ni directo, requiere de 
la creación personal. La memorización es, en este caso, una derivación del ejercicio de la sistematización creativa. La memorización de los conceptos clave, no es tarea inmediata y directa del trabajo de los sujetos, los conceptos se fijan como resultado de la sistematización del pensamiento, gracias a un sinnúmero de situaciones y tareas previstas que así la propician.

> La comunicación en su relación intrínseca con la actividad y su valor como procesos primigenios del desarrollo. La generalización como proceso dentro de la comunicaciónla actividad. El centro del programa de aprendizaje es precisamente la disección del proceso de comunicación, el análisis de su evolución desde los orígenes hasta la aparición de las palabras. La actividad de razonamiento está dirigida al estudio de dicho proceso, razón por la cual se entreteje con él naturalmente, tal como debiera corresponder, en su función, a la asignatura Lengua Materna en cualquier idioma.

> La aparición de neoformaciones psicológicas (conciencia del idioma) o su perfeccionamiento como indicador básico del desarrollo, más que la solución exitosa de determinadas tareas-problemas -aunque estas se utilizan como recursos importantes para la movilización del aprendizaje. Las neoformaciones tienen la propiedad de estructurar y reestructurar la personalidad, y por ende, de cambiar su dinámica. En el enfoque histórico cultural, la estructura y la función se asumen como una unidad dialéctica (la estructura-función). El cambio que ocurre con la aparición de la neoformación es propiamente un salto cualitativo en el desarrollo. La conciencia lingüística constituye uno de los grandes hitos del desarrollo de la personalidad y es base para el desarrollo de la conciencia humana en general. Las explicaciones anteriores sirven al análisis de este último punto.

Las experiencias humanistas de corte existencial, por ejemplo, resaltan el papel de los intercambios personales (de la empatía, entre otros procesos), pero esto es insuficiente para un histórico culturalista, porque significa -en su terminología- recortar la vivencia de la situación social del desarrollo, siempre anclada en la cultura. Los programas de desarrollo según el primer enfoque no ponderan de manera orgánicamente explícita la cultura y tampoco la historia. Otro ejemplo, es el de aquellas concepciones que destacan el papel del 
Otro, del grupo de coetáneos y de la ayuda, lo que es también insuficiente para ser declarada histórico culturalista.

De la misma manera, algunos autores tratan de asociar el enfoque histórico cultural con las competencias, de moda gracias al capitalismo cultural que trata de imponer un modelo hegemónico de pensamiento. Esta moda pondera aquellas habilidades que tienen que ver particularmente con la eficiencia de la empresa en ese sistema social. Amén del doble significado que tiene en nuestro idioma el término competencia, me preocupa que en su utilización no se da cuenta explícita de su visión pragmática y conductista del ser humano, que no busca la unidad de sentido en la persona declarando su centro en la personalidad sino en su fraccionamiento -inventario de competencias-, que hacen perder de vista la unidad de sentido sustituyéndola por la unidad de correlación, por la unidad estadística.

Además, su propuesta en los sistemas de evaluación y acreditación de programas de enseñanza persigue una marcada homogeneización de procesos y fenómenos de esencia heterogénea, lo que también intenta aniquilar el concepto de persona, de personalidad, por esencia variopinta -aunque guarden características generales- y unitarias gracias al sentido que es el que orienta su integración y desarrollo. No adición, no homogeneidad.

Tampoco hay que negar su ocurrencia y desarrollo, las competencias pueden ser consideradas como casos particulares de habilidades aplicables a instituciones -empresascon determinados fines de mercado y de torneos deportivos o de otra naturaleza. Homo habilis fue la denominación que la antropología dio al ser humano que dominó el desarrollo de sus habilidades en el proceso de la antropogénesis. Respeto esta historia. El concepto de competencia (D. McClelland 1961 y 1973, entre otros) surge para exponer una finalidad específica del comportamiento, que deviene general en los sistemas capitalistas. De aquí que considere incompatible la esencia de la perspectiva de las competencias con la perspectiva histórico cultural acerca del desarrollo humano. Hacerlas equivaler sería un acto eminentemente de reduccionismo.

He destacado hasta aquí las condiciones que considero necesario ponderar y mantener consecuentemente en la práctica, para ser considerado un autor o autora histórico culturalista. Mientras que en el debate acerca de las condiciones que considero suficientes, 
quisiera solo recalcar que no se trata de inventariar un sigma de condiciones, sino de captar el estilo, el ánima de su ideario, en definitiva: su esencia. A esta altura de la lectura podemos retomar la idea de que la asunción de uno, dos o tres conceptos vigotskyanos no nos acerca forzosamente, sino que nos puede alejar de este autor.

\section{Sobre el futuro del enfoque}

El enfoque histórico cultural, lejos de pretender perpetuarse como sistema conceptual cerrado -lo que sería contrario a su planteamiento esencial- procura principalmente ordenar el pensamiento crítico del investigador, desde el punto de vista gnoseológico-ético. Son la historia y la cultura el hilo de Ariadna de tal ordenamiento. Persigue la concepción compleja del desarrollo humano, a la que subordina su aparato conceptual. Para estos fines, nada mejor que crear macroconceptos, es el caso de: situación social del desarrollo, mediación, vivencia, ley genética, zona de desarrollo, entre otros. Todos susceptibles de ser utilizados en diversas disciplinas, de ser considerados como conceptos-base para la interdisciplina, la transdisciplina. Siguiendo el razonamiento vigotskyano: no valen tanto las disyuntivas (social o biológico, cultural o natural, objetividad o subjetividad, externo o interno, cognitivo o afectivo, educación o maduración, estructura o dinámica, parte o todo, cognitivo o afectivo, actividad o comunicación, fenómeno o esencia, entre otros debates conocidos), sino las integraciones, la composición múltiple del objeto de estudio. Lo que se completa con el permanente sentido histórico y cultural del análisis. Es por todo esto, que el enfoque se percibe con anclaje en la realidad, lejos de toda metafísica. Adaptable a las contingencias de la vida en sociedad, en sus diferentes contextos, sobre todo en aquellos que exigen una mejor educación y un elevado desarrollo humano.

Por tales características, aprecio que este enfoque está llamado a desempeñar un papel crucial en el desarrollo futuro de la psicología, de la pedagogía y otras ciencias sociales. Han ocurrido numerosas fusiones suyas -sobre todo reduccionistas-, por ejemplo con el cognitivismo y el constructivismo. Así es el curso de la historia.

No obstante, debieran sobrevenir fusiones superiores, donde sea verdadero cimiento, que sustente una nueva concepción del desarrollo humano, integrando lo mejor de cada corriente de pensamiento, sin pretender un pensamiento único. Aunque quedara subsumido, 
pero esencialmente presente orientado a la episteme, la ética de cada idea, de cada práctica. Ese día está por llegar y tal vez el camino sea largo y espinoso, pues todavía se prefiere trabajar con ideas más simples y empiristas, lo que es obra del pragmatismo, del positivismo que ha imperado en las ciencias y en las profesiones hasta el día de hoy. He estado trabajando en la Cátedra L. S. Vigotsky de la Facultad de Psicología de la Universidad de La Habana, el problema de la elaboración de la intertextualidad de los distintos enfoques con el enfoque histórico cultural. El problema radica aquí en la construcción de los intertextos y en los principios de su construcción (Fariñas, 2006, 2008 y 2009) ${ }^{15}$.

La sociedad tiene que cambiar para que cambie la ciencia. Pero podemos adelantar el camino para ese momento, que requerirá de una concepción más cabal y profunda del desarrollo humano. "Nuestra ciencia no podía ni puede desarrollarse en la vieja sociedad. Ser dueños de la verdad sobre la persona...es imposible mientras la humanidad no sea dueña de la verdad sobre la sociedad y de la sociedad" (Vigotsky, 2001, p. 475).

\section{Conclusiones}

La mera consideración de los conceptos y principios planteados por Vigotsky es insuficiente a fin comprender la esencia de sus planteamientos. Para estudiar su obra evadiendo el reduccionismo, el instrumentalismo es preciso partir de la comprensión de su idiosincrasia, filosofía y lógica de pensamiento. Las metáforas empleadas por él constituyen uno de sus principales recursos para ilustrar el giro que debía dar el pensamiento científico en la consecución de un punto de vista complejo y dialéctico para el estudio del desarrollo humano. Es de vital importancia la comprensión de la denominación -puesto que aparecen varias- que Vigotsky le diera al enfoque, a fin de no tergiversar la comprensión de su obra. La denominación de enfoque histórico cultural se debe a que la comprensión y explicación del desarrollo se produce a través del análisis de estas dos dimensiones, a la vez condicionantes generales del desarrollo humano. La concepción histórico culturalista no fue construida como una generalización lineal y directa de la práctica (inducción) en el sentido

15 En estos trabajos propongo una vía de acción integradora del enfoque histórico cultural, en los planos intradisciplinario, interdisciplinario y transdisciplinario. 
empirista, sino de la elaboración de una plataforma conceptual crítica, cuyo referente principal fue la práctica (método de ascensión de lo abstracto a lo concreto pensado), a la que retornó permanentemente gracias a la programación de intervenciones estimuladoras (orientación, ayuda, asesoramiento) en el desarrollo. La mayoría de las teorías del desarrollo humano, lo que hacen es constatar el desarrollo, no intervenir en este. El enfoque histórico cultural hizo énfasis en el saber humanístico, cualquiera que fuera el contenido del conocimiento al que se dirigiera su experimentación.

\section{REFERENCIAS}

Aidarova, Lada. (1969). El niño, el lenguaje y el saber humanístico (en ruso). Pedagogía Soviética, (6), Moscú.

Aidarova, Lada. (1979). Problemas psicológicos de la enseñanza de la lengua materna a niños de edad escolar (en ruso). Moscú: Ed. Pedagogía.

Bruner, Jerome. (1997). La educación, puerta de la cultura. Madrid: Ed. Visor.

Davidov, Vasili Vasielevich. (1981). Tipos de generalización en la enseñanza. La Habana: Ed. Pueblo y Educación.

Elkonin, Danil (1972). Hacia una periodización del desarrollo psicológico en la infancia (en ruso). Cuestiones de Psicología, (4), Moscú.

Fariñas, Gloria. (2005). Psicología, educación y sociedad. La Habana: Ed. Félix Varela.

Fariñas, Gloria. (2008) Debates teórico metodológicos de la Cátedra L. S. Vigotsky. En Educación, cultura y desarrollo humano, (2), Sao Paulo: Ed .Terceira Margem.

Fariñas, Gloria. (2009) Debates teórico metodológicos de la Cátedra L. S. Vigotsky. En Educación, cultura y desarrollo humano, (3), Sao Paulo: Ed .Terceira Margem.

Fariñas, Gloria y García, David. (2006). De la paradoja de Russell a la complejidad del desarrollo humano en busca de los principios orientadores. En Educación, cultura y desarrollo humano, (1), Sao Paulo: Ed .Terceira Margem.

Geertz, Clifford. (2003). Impacto del concepto de cultura en el concepto de hombre. En colectivo de autores. Antropología social. La Habana: Ed. Félix Varela.

Marx, Carlos y Engels, Federico. (1961). Obras Escogidas. Tomo II. Moscú: Ed. Progreso.

McClelland, David. (1961). The Achieving Society. Nueva Jersey: Princeton, Van Nostrand. 
McClelland, David. (1973).Testing for Competence Rather Than for Intelligence. En: American Psychologist, (28), 1-14.

Mecheriakov, Boris. (1998). Análisis lógico semántico de la concepción de Vigotsky. Samara: Editorial de la Universidad Pedagógica Estatal de Samara (en ruso).

Rogers, Carl. (1993). Libertad y creatividad en la educación (en la década de los ochentas). Buenos Aires: Ed. Paidós.

Vygotski, Lev. (1987). Historia del desarrollo de las funciones psíquicas superiores. La Habana: Ed. Científico Técnica.

Vygotski, Lev. (1982a). El significado histórico de la crisis de la psicología. En Obras Escogidas. Tomo I (pp. 190-400). Moscú: Ed. Pedagogía (en ruso).

Vygotski, Lev. (1982b). Pensamiento y lenguaje. En Obras Escogidas. Tomo II en ruso. (pp.8-290). Moscú: Ed. Pedagogía (en ruso).

Vygotski, Lev. (2001). Psicología Pedagógica. Un curso breve. Buenos Aires: Ed. Grupo Aique S. A. 